\title{
Numerically Intersecting Algebraic Varieties via Witness Sets
}

\author{
Jonathan D. Hauenstein* Charles W. Wampler ${ }^{\dagger}$
}

April 19, 2012

\begin{abstract}
The fundamental construct of numerical algebraic geometry is the representation of an irreducible algebraic set, $A$, by a witness set, which consists of a polynomial system, $F$, for which $A$ is an irreducible component of $\mathcal{V}(F)$, a generic linear space $\mathcal{L}$ of complementary dimension to $A$, and a numerical approximation to the set of witness points, $\mathcal{L} \cap A$. Given $F$, methods exist for computing a numerical irreducible decomposition, which consists of a collection of witness sets, one for each irreducible component of $\mathcal{V}(F)$. This paper concerns the more refined question of finding a numerical irreducible decomposition of the intersection $A \cap B$ of two irreducible algebraic sets, $A$ and $B$, given a witness set for each. An existing algorithm, the diagonal homotopy, computes witness point supersets for $A \cap B$, but this does not complete the numerical irreducible decomposition. In this paper, we use the theory of isosingular sets to complete the process of computing the numerical irreducible decomposition of the intersection.
\end{abstract}

Key words and phrases. witness point, witness set, diagonal homotopy, intersection, irreducible algebraic set, deflation, isosingular set, isosingular point, numerical algebraic geometry, polynomial system

2010 Mathematics Subject Classification. Primary 65H10; Secondary 13P05, 14Q99, $68 \mathrm{~W} 30$.

\section{Introduction}

This paper concerns the computation of the intersection, $A \cap B$, of two irreducible algebraic sets, $A$ and $B$. Since the intersection of two general algebraic sets can be broken down into a collection of pair-wise intersections of the irreducible components of those sets, with some membership testing to eliminate multiple appearances of components or strict containment of one in another, the pair-wise intersection of irreducibles is seen to be the crux of a general intersection capability. The method of this paper fits into the framework of numerical algebraic geometry, a term coined in 30. The area relies on polynomial homotopy algorithms, often called polynomial continuation, to reliably and efficiently compute numerical approximations to the solutions of systems of polynomial equations. Overviews of the field may be found in

\footnotetext{
*Department of Mathematics, Mailstop 3368, Texas A\&M University, College Station, TX 77843 (jhauenst@math.tamu.edu, www.math.tamu.edu/ jhauenst). This author was supported by Texas A\&M University, the Mittag-Leffler Institute, and NSF grants DMS-0915211 and DMS-1114336.

${ }^{\dagger}$ General Motors Research and Development, Mail Code 480-106-359, 30500 Mound Road, Warren, MI 48090 (Charles.W.Wampler@gm.com, www.nd.edu/ cwample1) This author was supported by NSF grant DMS-0712910 and the Mittag-Leffler Institute.
} 
[31, 32. The basics of polynomial continuation for finding isolated solutions are covered in [15, 16, 18, 31].

In numerical algebraic geometry, an irreducible algebraic set, $A \subset \mathbb{C}^{N}$, is represented by a witness set, $W$, of the form

$$
W=\{F, \mathcal{L}, \mathcal{L} \cap A\},
$$

where $F: \mathbb{C}^{N} \rightarrow \mathbb{C}^{M}$ is a polynomial system such that $A$ is an irreducible component of

$$
\mathcal{V}(F):=\left\{x \in \mathbb{C}^{N} \mid F(x)=0\right\},
$$

$\mathcal{L}$ is a generic linear space of complementary dimension to $A$ (i.e., $\operatorname{dim} \mathcal{L}=N-\operatorname{dim} A$ ), and hence, $\mathcal{L} \cap A \subset \mathbb{C}^{N}$ is a set of $\operatorname{deg} A$ isolated points. Of course, in numerical work, the points $\mathcal{L} \cap A$ are not known exactly but rather are represented by numerical approximations. We call $W=\{F, \mathcal{L}, \mathcal{L} \cap A\}$ a witness set; it contains a witness system, $F$, and a witness point set, $\mathcal{L} \cap A$.

One of the fundamental operations of numerical algebraic geometry is the computation of a numerical irreducible decomposition, which finds a witness set for each irreducible component of $\mathcal{V}(F)$. The first algorithm for computing a numerical irreducible decomposition appeared in [26, with subsequent improvements in 2, 27, 28, 29. Let the pure $i$-dimensional component of $\mathcal{V}(F)$ be denoted $Z_{i}$ (possibly empty for some $i$ ), which decomposes into a finite number, $n_{i}$, of distinct irreducible components $Z_{i j}$; that is,

$$
\mathcal{V}(F)=\bigcup_{i=0}^{\operatorname{dim} \mathcal{V}(F)} Z_{i}, \quad Z_{i}=\bigcup_{j=1}^{n_{i}} Z_{i j}
$$

All of the existing algorithms for the irreducible decomposition first compute witness supersets, $\widehat{W}_{i}=\left\{F, \mathcal{L}_{i}, \widehat{S}_{i}\right\}$, where $\mathcal{L}_{i}$ is a generic linear space of codimension $i$ and each witness point superset $\widehat{S}_{i}$ is a finite set of points such that $\left(\mathcal{L}_{i} \cap Z_{i}\right) \subset \widehat{S}_{i} \subset \mathcal{V}(F)$. Let us denote the local dimension of algebraic set $A$ at $z$, as $\operatorname{dim}_{z} A$. To winnow a witness point set from a witness point superset, a filtering algorithm culls out the junk points $J_{i}=\left\{x \in \widehat{S}_{i} \mid \operatorname{dim}_{x} \mathcal{V}(F)>i\right\}$. For points with a low multiplicity bound (obtained as the number of homotopy paths leading to it), this filtering is most efficiently done using a local dimension test [2]; otherwise, homotopy membership tests [27. determine if $x$ is in any component of higher dimension, these having been determined already in sequential order starting at the top dimension. Then the remaining points, $S_{i}=\widehat{S}_{i} \backslash J_{i}$, are partitioned using monodromy [29] and trace tests [28] to form the witness point sets for the irreducible components, $S_{i j}=\mathcal{L}_{i} \cap Z_{i j}$, thereby forming the witness sets $W_{i j}=\left\{F, \mathcal{L}_{i}, S_{i j}\right\}$. It is possible to do this at each dimension independently, but it is generally more efficient to descend sequentially through all possible values of $i$ using a cascade approach [9, 21].

Once a witness set for an irreducible algebraic set $A$ is known, one can subsequently apply other operations of numerical algebraic geometry to it. For example, it is possible to test if a given point is in $A$ and, if $A$ is positive dimensional, to compute a scattered sampling of points numerically in $A$. One can also answer many questions about $A$, such as its degree and multiplicity structure. Also, given witness sets for two irreducible algebraic sets, one can test if either is contained in the other.

One operation that is incomplete in numerical algebraic geometry is intersection. That is, given witness sets, $\left\{F_{A}, \mathcal{L}_{A}, \mathcal{L}_{A} \cap A\right\}$ and $\left\{F_{B}, \mathcal{L}_{B}, \mathcal{L}_{B} \cap B\right\}$, for irreducible algebraic sets $A$ and $B$, we wish to compute the intersection $A \cap B$ as represented by a numerical irreducible decomposition. This would consist of a collection of witness sets, one for each irreducible component of 
$A \cap B \subset \mathbb{C}^{N}$. Similar to the case described above for computing a numerical irreducible decomposition of $\mathcal{V}(F)$, let $Z_{i}$ be the pure $i$-dimensional component of $A \cap B$, which has an irreducible decomposition $Z_{i}=\cup_{j=1}^{n_{i}} Z_{i j}$. For each dimension $i$ where $i$-dimensional components $Z_{i j}$ of $A \cap B$ could exist, i.e., for $\max \{0, \operatorname{dim} A+\operatorname{dim} B-N\} \leq i \leq \min \{\operatorname{dim} A, \operatorname{dim} B\}$, existing diagonal homotopy methods compute a finite witness point superset, $\widehat{S}_{i}$, such that $\left(\mathcal{L}_{i} \cap Z_{i}\right) \subset \widehat{S}_{i} \subset(A \cap B)$, where $\operatorname{dim} \mathcal{L}_{i}=N-i$. Diagonal methods work by duplicating the variables from, say, $x \in \mathbb{C}^{N}$, to $(x, y) \in \mathbb{C}^{2 N}$, and finding subsets of $A \times B=\{(x, y) \mid x \in A, y \in B\}$ such that $x=y$. The first such algorithm [23] uses a cascade approach that doubles the number of variables, while a subsequent reformulation [24] in intrinsic coordinates reduces the number of variables.

The computation of the witness point supersets $\widehat{S}_{i}$ is a crucial first step, but to complete the numerical irreducible decomposition of $A \cap B$, one must eliminate any junk points in the superset to obtain a witness point set for $Z_{i}$ and then break these set into witness point sets for the irreducible components $Z_{i j}$. As we shall see, the local dimension test used for eliminating junk in the standard numerical irreducible decomposition problem no longer applies to the pairwise intersection problem. Furthermore, for each irreducible component $Z_{i j}$, the witness set requires a witness system $F_{i j}$ such that $Z_{i j}$ is one of its irreducible components. In some cases, $F_{i j}=\left\{F_{A}, F_{B}\right\}$ suffices, but as we shall illustrate below, this is not true in general.

The purpose of this paper is to complete the diagonal approach for computing a numerical irreducible decomposition of a pair-wise intersection. To do so, we provide an alternative approach to filtering out junk points and show how to generate the witness systems $F_{i j}$ to complete the irreducible witness sets. The necessary tools are provided by the recently developed theory of isosingular sets [10]. The existing diagonal homotopy suffices as is when all the irreducible components of $A \cap B$ are also irreducible components of $\mathcal{V}\left(F_{A}, F_{B}\right)$. On the contrary, suppose $C$ is some irreducible component of $A \cap B$ which is not an irreducible component of $\mathcal{V}\left(F_{A}, F_{B}\right)$. Then, $C$ may or may not be an isosingular set with respect to $\left\{F_{A}(x), F_{B}(x)\right\}$, but as we shall prove, it is always isomorphic to the intersection of $\mathcal{V}(x-y)$ with an isosingular set of the related system $\left\{F_{A}(x), F_{B}(y)\right\}$. Using this fact, one can compute a witness set for $C$ and, in this way, complete the numerical irreducible decomposition of $A \cap B$.

While the completion of the diagonal intersection via isosingular theory is the main purpose of this paper, a secondary contribution is to present a simplified formulation of the diagonal homotopy. This formulation is advantageous in the construction of a working computer algorithm.

\section{Insufficiency of the diagonal homotopy}

Although the existing diagonal homotopies compute witness point supersets for an intersection, this is not enough to produce true witness sets and the irreducible decomposition. As mentioned above, there may exist an irreducible component $C \subset A \cap B$ that is not an irreducible component of the combined system $\left\{F_{A}, F_{B}\right\}$. A few simple examples follow to illustrate this fact.

Example 1.0.1 Consider the polynomial

$$
f(x, y, z)=(x-1)\left(x^{2}+y^{2}+z^{2}-4\right)\left((x-2)^{2}+y^{2}+z^{2}-4\right) .
$$

From the factored form of $f$, one sees that $\mathcal{V}(f)$ factors into a plane $P_{1}:=\mathcal{V}(x-1)$ and two spheres, say $S_{1}$ and $S_{2}$. A numerical irreducible decomposition applied to $\mathcal{V}(f)$ will produce three witness sets, with $f$ as the polynomial system appearing in each. Starting with the witness sets for $S_{1}$ and $S_{2}$, one may then proceed, using a diagonal homotopy [23, 24], to find points on 
the intersection $S_{1} \cap S_{2}$. The diagonal homotopy constructs a general plane, $P_{2}$, and computes two general points, say $\left\{w_{1}, w_{2}\right\}=\left(S_{1} \cap S_{2}\right) \cap P_{2}$.

It happens that $S_{1} \cap S_{2}$ is a circle that lies inside $P_{1}$, so it is not an irreducible component of $\mathcal{V}(f)$. Accordingly, $\left\{f, P_{2},\left\{w_{1}, w_{2}\right\}\right\}$ is not a witness set for $S_{1} \cap S_{2}$. To get a witness set, a symbolic operation, trivial in this case, is needed to produce a witness system, say

$$
G(x, y, z)=\left\{x^{2}+y^{2}+z^{2}-4,(x-2)^{2}+y^{2}+z^{2}-4\right\},
$$

so that a witness set for $S_{1} \cap S_{2}$ is $\left\{G, P_{2},\left\{w_{1}, w_{2}\right\}\right\}$.

It is easy to see that $S_{1} \cap S_{2}$ is in the singular set of $\mathcal{V}(f)$, and one might suppose that this would always happen for examples where the diagonal homotopy fails to yield a true witness set. However, the next example shows that this is not always the case.

Example 1.0.2 Consider the polynomials $f_{1}(x, y, z)=x$ and $f_{2}(x, y, z)=x y$. The algebraic set $\mathcal{V}\left(f_{1}\right)$ has a single irreducible component, the plane $V_{1}:=\mathcal{V}(x)$, while $\mathcal{V}\left(f_{2}\right)$ has two irreducible components, $V_{1}$ and $V_{2}:=\mathcal{V}(y)$. The intersection of $V_{1}$ and $V_{2}$ is a line: $V_{1} \cap V_{2}=\mathcal{V}(x, y)$. Meanwhile, the solution set of the system $\left\{f_{1}, f_{2}\right\}$ is the plane $V_{1}$, and each point in $V_{1} \cap V_{2}$ is a smooth point of $V_{1}$. So although a diagonal homotopy will produce a general point, say $p$, on the line $V_{1} \cap V_{2}$, it again fails to produce a true witness set directly because we do not have a witness system. Notice also that a local dimension test will say that $p$ lies on a two-dimensional component of $\mathcal{V}\left(f_{1}, f_{2}\right)$. Since this does not properly indicate the dimension of the line $V_{1} \cap V_{2}$ which $p$ witnesses, we see that the local dimension test cannot reliably distinguish between junk points and witness points in a witness point superset produced by a diagonal homotopy.

One of the main contributions of this paper is to give a numeric-symbolic method that takes the witness point supersets generated by a diagonal homotopy and creates witness sets. As we see in the examples above, this means that for each component found in a diagonal homotopy, we may need to modify the given polynomials to produce a witness system, the first entry in a witness set. We also need a replacement for the local dimension test in order to detect and expunge junk points from witness point supersets.

\section{Deflation and isosingular sets}

Deflation refers to procedures for reducing the multiplicity of an isolated solution point or reducing the generic multiplicity of an irreducible component. Originally, the methods were aimed solely at isolated solution points. Suppose that $x^{*} \in \mathbb{C}^{N}$ is an isolated point in $\mathcal{V}(F)$ having multiplicity, $\mu$, greater than one. Heuristic methods using derivatives and determinants in [19, 20] lead to the Leykin-Verschelde-Zhao deflation method [14 that constructs an extended system $\{F(x), G(x, \lambda)\}$ such that $\mathcal{V}(F, G)$ has an isolated point $\left(x^{*}, \lambda^{*}\right)$ of multiplicity at most $\mu-1$. Letting $\{F(x), G(x, \lambda)\}$ and $\left(x^{*}, \lambda^{*}\right)$ play the roles of the original $F$ and $x^{*}$, one may repeat the construction iteratively until one arrives at a system that has a nonsingular root corresponding to $x^{*}$. Dayton and Zeng [5] used a construction called a Macaulay matrix to help understand the number of iterations required to reach nonsingularity, and this lead to a higherorder deflation method in [13] and the closedness-subspace method [33. (On another track, a symbolic deflation method was presented in [12.) It was observed in 31] that the LVZ deflation method, when applied to a witness point isolated by slicing an irreducible component with a generic linear space, yields a way of treating nonreduced solution components. All of these methods produce a deflated system in a larger number of variables than the original system, 
and the growth can be considerable when multiple iterations are required. The extra variables, $\lambda$, are related to vectors in the null space of the Jacobian matrix of $F$.

None of these deflation methods apply directly to a nonsmooth solution point of $\mathcal{V}(F)$ or a point on an embedded component. This does not matter in the standard numerical irreducible decomposition, because a generic linear space of complementary dimension meets an irreducible component in a generic point. But as shown by Example 1.0.1 in the pair-wise intersection problem it may happen that every point in the intersection is a nonsmooth point of the solution set of the polynomial system at hand.

Isosingular theory [10] uses a strong deflation operation that is related to the weak deflation method of [14. Let $J F(x)$ be the $M \times N$ Jacobian matrix of partial derivatives of $F: \mathbb{C}^{N} \rightarrow \mathbb{C}^{M}$, and suppose $x^{*} \in \mathcal{V}(F) \subset \mathbb{C}^{N}$. Define dnull $\left(F, x^{*}\right):=N-\operatorname{rank} J F\left(x^{*}\right)$, the dimension of the right null space of the Jacobian matrix evaluated at $x^{*}$. In essence, the polynomial system $G(x, \lambda)$ appended to $F$ by weak deflation is a necessary condition for dnull $(F, x) \geq \operatorname{dnull}\left(F, x^{*}\right)$, whereas strong deflation appends a system that enforces a necessary and sufficient condition. The details of the constructions are not required here.

There is a determinantal version of strong deflation that does not expand the variable set. In this version, the equations that are appended are the determinants of the $(N-d+1) \times(N-d+1)$ minors of $J F(x)$, where $d=$ dnull $\left(F, x^{*}\right)$. Let $D\left(F, x^{*}\right)$ denote the new polynomial system derived by appending these determinants to $F(x)$, and let $D^{k}\left(F, x^{*}\right)$ be the result of iterating this procedure $k$ times. By convention, $D^{0}\left(F, x^{*}\right)=F(x)$. At every stage, $x^{*}$ is still a solution of $D^{k}\left(F, x^{*}\right)=0$. Then, given $F$ and $x^{*} \in \mathcal{V}(F)$, one obtains an infinite sequence of integers, $d_{k}\left(F, x^{*}\right)$, called the deflation sequence of $x^{*}$ with respect to $F$, defined as

$$
d_{k}\left(F, x^{*}\right)=\operatorname{dnull}\left(D^{k}\left(F, x^{*}\right)\right), \quad k=0,1, \ldots
$$

This sequence is the same as the Thom-Boardman singularity type of $x^{*}$ in the theory of smooth mappings [1, 4]. For the purposes of this paper, the determinantal form of strong deflation is preferred over other forms given in [10, that introduce auxiliary null-space variables $\lambda$. In particular, when auxiliary variables are introduced, the original point $x^{*}$ is a projection of the final point $\left(x^{*}, \lambda^{*}\right)$, which complicates matters when $x^{*}$ is a point on some positive dimensional set that we wish to subject to further operations.

Determinantal strong deflation can be viewed as a purely symbolic process, but when $x^{*}$ is given numerically, it becomes a numeric-symbolic process. This is because the derivatives and the determinants are formed symbolically, but at each deflation step, the size of the minors entering the determinants is an integer found by evaluating the rank of the Jacobian matrix numerically using a singular value decomposition. It is assumed that the point $x^{*}$ is known accurately enough so that the sequence of ranks is decided correctly. For this purpose, it may be important to use extended precision arithmetic to compute $x^{*}$ and the singular values.

Among all the points in $\mathcal{V}(F)$, there is some subset of points that have the same deflation sequence as $x^{*}$. This set may have several irreducible components, one of which must contain $x^{*}$. The algebraic closure of this component is called the isosingular set of $x^{*}$ with respect to $F$, written $\operatorname{Iso}_{F}\left(x^{*}\right)$. As the deflation sequence is the same on a dense Zariski-open subset of any irreducible algebraic set, say $Z$, we may define $d_{k}(F, Z)=d_{k}\left(F, x^{*}\right)$ and $\operatorname{Iso}_{F}(Z)=\operatorname{Iso}_{F}\left(x^{*}\right)$, where $x^{*}$ is any generic point of $Z$. An isosingular set with respect to $F$ is defined to be any set that is invariant under the function $\operatorname{Iso}_{F}$, that is, any set $Z$ such that $Z=\operatorname{Iso}_{F}(Z)$. For easy reference below, we list a few of the properties of isosingular sets in the following theorem.

Theorem 2.0.3 (Isosingular Properties) Suppose $Z$ is an isosingular set with respect to polynomial system $F$. Then all of the following hold. 
1. The deflation sequence of $Z$ is nonincreasing and nonnegative.

2. There exists a finite $k^{*}$ such that the deflation sequence of $Z$ stabilizes to $d_{k}(F, Z)=$ $d_{k^{*}}(F, Z)=\operatorname{dim} Z$ for all $k \geq k^{*}$. We call $k^{*}$ the isosingular stabilization index of $Z$.

3. For $k^{*}$ as in Item 2, $Z$ is a generically reduced 1 irreducible component of $D^{k^{*}}(F, Z)$.

4. Irreducible components are isosingular.

5. For any polynomial system $F, \mathcal{V}(F)$ has a finite number of isosingular sets.

Proof. All of these claims are proven in [10].

It is important to note that there exists a computable test to determine when the stabilization value $k^{*}$ of Item 2 is reached [10].

Item 3 will have important consequences in our treatment of the pair-wise intersection problem. As described in Section 4 this gives us a method of constructing a polynomial system that has the set as an irreducible component. This is the witness system that we need for the first entry in a witness set for the component.

\section{Simplified diagonal homotopy}

The algorithms of [23, 24] use cascading diagonal homotopies to compute witness point supersets for the intersection of two algebraic varieties. Unfortunately, the formulations presented in these papers are more complicated than necessary due to an overuse of randomization. In this section, we present simplified formulations of diagonal homotopies, one extrinsic as in [23] and one intrinsic as in 24 .

Given two irreducible algebraic sets $A, B \subset \mathbb{C}^{N}$, our objective is to find $A \cap B$. The basic maneuver in a diagonal homotopy is to shift the focus to the set

$$
X=A \times B=\left\{(x, y) \in \mathbb{C}^{2 N} \mid x \in A, y \in B\right\}
$$

and find within this set the points where $x=y$. That is, defining

$$
\Delta=\left\{(x, x) \mid x \in \mathbb{C}^{N}\right\}=\mathcal{V}(x-y) \subset \mathbb{C}^{2 N},
$$

we seek the components of $X \cap \Delta$. This suffices because the irreducible components of $X \cap \Delta$ are isomorphic to those of $A \cap B$. To be precise, let $Z_{i j}$ be the irreducible components of $A \cap B$ in the manner of $(3)$, and let $\mathcal{Z}_{i j}$ be those for $X \cap \Delta$. Then, defining the maps

$$
\begin{gathered}
\pi_{\Delta}: \Delta \rightarrow \mathbb{C}^{N}:(x, x) \mapsto(x), \\
\pi_{\Delta}^{-1}: \mathbb{C}^{N} \rightarrow \Delta:(x) \mapsto(x, x),
\end{gathered}
$$

it is clear that subscripts can be assigned such that $Z_{i j}=\pi_{\Delta}\left(\mathcal{Z}_{i j}\right)$ and $\mathcal{Z}_{i j}=\pi_{\Delta}^{-1}\left(Z_{i j}\right)$ for every irreducible component. Consequently, the new objective is to find witness sets for the irreducible components, $\mathcal{Z}_{i j}$, of $X \cap \Delta$.

We assume that at the outset $A$ and $B$ are given in terms of witness sets $W_{A}=\left\{F_{A}, \mathcal{L}_{A}, S_{A}\right\}$ and $W_{B}=\left\{F_{B}, \mathcal{L}_{B}, S_{B}\right\}$, respectively. That is, let $F_{A}: \mathbb{C}^{N} \rightarrow \mathbb{C}^{n_{a}}$ and $F_{B}: \mathbb{C}^{N} \rightarrow \mathbb{C}^{n_{b}}$ be

\footnotetext{
${ }^{1}$ An irreducible component $Z$ of $\mathcal{V}(F)$ is said to be generically reduced if there is a nonempty Zariski-open subset $U \subset Z$ such that each point $u \in U$ has multiplicity one with respect to $F$.
} 
polynomial systems, and suppose $A \subset \mathcal{V}\left(F_{A}\right) \subset \mathbb{C}^{N}$ and $B \subset \mathcal{V}\left(F_{B}\right) \subset \mathbb{C}^{N}$ are irreducible components with $\operatorname{dim} A=a>0$ and $\operatorname{dim} B=b>0$. By Items 3 and 4 of Theorem 2.0.3, we can assume without loss of generality that $A$ and $B$ are generically reduced components of $F_{A}^{-1}(0)$ and $F_{B}^{-1}(0)$, respectively. For a set of polynomials $p=\left\{p_{1}, \ldots, p_{n}\right\}$, let $\mathcal{R}(p, k)$ be a new set of $k$ polynomials, each one a random linear combination of $p_{1}, \ldots, p_{n}$. By Theorem 13.5.1, Item (2) in [31, p.243], we may "square up" $F_{A}$ and $F_{B}$ by replacing them with $\mathcal{R}\left(F_{A}, N-a\right)$ and $\mathcal{R}\left(F_{B}, N-b\right)$, resp., so after renaming these, we have $F_{A}: \mathbb{C}^{N} \rightarrow \mathbb{C}^{N-a}$ and $F_{B}: \mathbb{C}^{N} \rightarrow \mathbb{C}^{N-b}$, with generically reduced, irreducible components $A$ and $B$, respectively.

We will also assume that $A \not \subset B$ and $B \not \subset A$ which can be verified using the witness sets $W_{A}$ and $W_{B}$ together with a homotopy membership test. By renaming if necessary, we can assume that $a \geq b$ and hence $\operatorname{dim} A \cap B<b$. Moreover, dimension counting reveals that any nonempty component of $A \cap B$ must have dimension at least $\kappa:=\max \{0, a+b-N\}$. Hence, our first objective will be to find witness point supersets for the pure $i$-dimensional components, $Z_{i}$, of $A \cap B$, for $\kappa \leq i \leq b-1$. By the discussion above, this is equivalent to finding witness point supersets for $\mathcal{Z}_{i}$, the pure $i$-dimensional components of $X \cap \Delta$.

To proceed, we introduce notation for the vector space of a set of polynomials. For polynomials $p_{1}, \ldots, p_{n}$, let $\left\langle p_{1}, \ldots, p_{n}\right\rangle$ be the vector space of linear combinations of $p_{1}, \ldots, p_{n}$; that is, each element of $\left\langle p_{1}, \ldots, p_{n}\right\rangle$ has the form

$$
a_{1} p_{1}+\cdots+a_{n} p_{n}, \quad\left(a_{1}, \ldots, a_{n}\right) \in \mathbb{C}^{n} .
$$

Accordingly, sets of $k$ elements from $\left\langle p_{1}, \ldots, p_{n}\right\rangle$ form a space

$$
\left\langle p_{1}, \ldots, p_{n}\right\rangle^{k}:=\underbrace{\left\langle p_{1}, \ldots, p_{n}\right\rangle \times \cdots \times\left\langle p_{1}, \ldots, p_{n}\right\rangle}_{k \text { times }},
$$

which has a parameterization as $\mathbb{C}^{k \times n}$. If we let $P=\left\{p_{1}, \ldots, p_{n}\right\}$, then the notations $\langle P\rangle$ and $\left\langle p_{1}, \ldots, p_{n}\right\rangle$ are taken to mean the same thing. The randomization operation $\mathcal{R}(P, k)$ means to pick at random a member of $\langle P\rangle^{k}$.

With this notation, Lemma 3.1 of 23 (a generalization of Theorem 13.5.1 of 31]) implies that there is a nonempty Zariski-open set $U_{i} \subset\langle x-y\rangle^{a+b-i}, \kappa \leq i \leq b$, such that for $D_{i} \in U_{i}$ :

1. any $i$-dimensional irreducible component $\mathcal{Z}_{i j}$ of $X \cap \Delta$ is also an irreducible component of $X \cap \mathcal{V}\left(D_{i}\right)$; and

2. for $k>i$, the $k$-dimensional irreducible components of $X \cap \Delta$ and $X \cap \mathcal{V}\left(D_{i}\right)$ are the same.

Furthermore, by Bertini's Theorem (see Theorem B.1 of [23]), we have that there is a nonempty Zariski-open subset $U^{\prime} \subset\langle x-y\rangle^{a+b-i} \times\langle x, y, 1\rangle^{i}, \kappa \leq i \leq b$, such that for $\left(D_{i}, L_{i}\right) \in U_{i}^{\prime}$ :

3. the points in $X \cap \mathcal{V}\left(D_{i}, L_{i}\right)$ that do not lie in $\Delta$ are isolated and nonsingular.

Pick a $\left(D_{i}, L_{i}\right)$ in $U_{i}^{\prime}$. Then, Items 1 and 2 tell us that the isolated points in $X \cap \mathcal{V}\left(D_{i}, L_{i}\right)$ that lie in $\Delta$ are, under the projection $\pi_{\Delta}$, a witness point set for the pure $i$-dimensional component $Z_{i}$ of $A \cap B$.

Because the intersection of a finite number of nonempty Zariski-open sets is still nonempty Zariski-open, there is a nonempty Zariski-open set $\widetilde{U}_{\kappa}$ in $\langle x-y\rangle^{a+b-\kappa}$ such that for $D_{\kappa} \in \widetilde{U}_{\kappa}$, choosing $D_{i}$ as the first $a+b-i$ elements of $D_{\kappa}, \kappa \leq i \leq b-1$, means that $D_{i}$ is in $U_{i}$, the sets where Items 1 and 2 hold. In this way, if we choose once and for all $D_{\kappa} \in\langle x-y\rangle^{a+b-\kappa}$ at random, then with probability one, we have also selected all the $D_{i}$ systems generically in 
the respective $U_{i}$. Similarly, we may choose one system of $(b-1)$ general linear equations $L_{b-1} \in\langle x, y, 1\rangle^{b-1}$ and use $i$ of these as the general linear system for $L_{i}$ in Item 3 above.

To be more explicit, for $i=\kappa, \kappa+1, \ldots, b-1$, let

$$
\begin{aligned}
D_{i} & =\left[\begin{array}{llll}
\delta_{1} & \delta_{2} & \cdots & \delta_{a+b-i}
\end{array}\right], \\
L_{i} & =\left[\begin{array}{llll}
\ell_{b-1} & \ell_{b-2} & \cdots & \ell_{1}
\end{array}\right] .
\end{aligned}
$$

For $i=\kappa, \kappa+1, \ldots, b-1$, define $\mathcal{E}_{i}: \mathbb{C}^{2 N} \rightarrow \mathbb{C}^{2 N}$ as

$$
\begin{aligned}
\mathcal{E}_{i}(x, y) & =\left\{F_{A}(x), F_{B}(y), D_{i}, L_{i}\right\} \\
& =\left\{F_{A}(x), F_{B}(y), \delta_{1}, \delta_{2}, \ldots, \delta_{a+b-i}, \ell_{i}, \ell_{i-1}, \ldots, \ell_{1}\right\}
\end{aligned}
$$

Notice that $\mathcal{E}_{i}$ and $\mathcal{E}_{i-1}$ differ only in that $\ell_{i}$ becomes $\delta_{a+b-i+1}$; all other polynomials in the list remain the same.

Since each $\delta_{i}$ is a special kind of linear while each $\ell_{i}$ is a general linear, the isolated solutions of $\mathcal{E}_{i}=0$ can be tracked in a parameter homotopy taking $\ell_{i}$ to $\delta_{a+b-i}$ to find the isolated solutions of $\mathcal{E}_{i-1}$. This is the key to setting up a cascade of homotopies to find witness supersets at every dimension. We will not prove the details here, as the arguments are essentially the same as those given in 23 for the original diagonal cascade method. However, in the next two subsections, we will write down the explicit homotopy formulations we use, one extrinsic and one intrinsic.

\subsection{Extrinsic formulation}

Define $S_{X}=\left\{(x, y) \mid x \in S_{A}, y \in S_{B}\right\}$, a set of $\operatorname{deg} X=(\operatorname{deg} A) \cdot(\operatorname{deg} B)$ points. We know that

$$
W_{X}=\left\{\left(F_{A}(x), F_{B}(y)\right), \mathcal{L}_{A} \times \mathcal{L}_{B}, S_{X}\right\}
$$

is a witness set for $X$. From this witness set, we can compute the intersection of $X$ with any linear subspace of complimentary dimension by setting up a homotopy that moves from $\mathcal{L}_{A} \times \mathcal{L}_{B}$ to the target linear subspace. Letting $L_{A}$ and $L_{B}$ be linear systems such that $\mathcal{L}_{A}=\mathcal{V}\left(L_{A}\right)$ and $\mathcal{L}_{B}=\mathcal{V}\left(L_{B}\right)$, a valid homotopy is $H_{X, i}: \mathbb{C}^{2 N} \times \mathbb{C} \rightarrow \mathbb{C}^{2 N}$ defined as

$$
H_{X, i}(x, y, t)=\left\{F_{A}(x), F_{B}(y), \gamma t\left[L_{A}(x) \quad L_{B}(y)\right]+(1-t)\left[D_{i}(x, y) \quad L_{i}(x, y)\right]\right\},
$$

where $\gamma$ is a random point in $\mathbb{C}$. The homotopy starts at $t=1$ with the points $S_{X}$ and tracks $\operatorname{deg} X$ solution paths as $t$ moves monotonically in $(0,1]$, approaching $t=0$ in the limit. For any $\gamma$ not lying on one of a finite number of rays from the origin in $\mathbb{C}$, the endpoints of these homotopy paths that lie on $\Delta$ form a witness point superset for dimension $i$. Computing all the intersections $X \cap \mathcal{V}\left(D_{i}, L_{i}\right), i=\kappa, \ldots, b-1$, in this way gives a witness point superset for every possible dimension.

Instead of computing each witness point superset in an independent homotopy starting from $W_{X}$, it is more efficient to organize a cascade of homotopies in which a witness point superset for dimension $i$ is computed starting with the results of the previous homotopy for dimension $(i+1)$. The cascade starts out by using $H_{X, b-1}(x, y, t)$ from 10 to get a witness point superset for dimension $(b-1)$ and then proceeds down the dimensions using the sequence of homotopies:

$$
H_{i+1, i}(x, y, t)=t \mathcal{E}_{i+1}(x, y)+(1-t) \mathcal{E}_{i}(x, y), \quad i=b-2, b-3, \ldots, \kappa .
$$


From (9), we may rewrite this as

$$
\begin{aligned}
H_{i+1, i}(x, y, t)=\left\{F_{A}(x), F_{B}(y)\right. & , \delta_{1}, \delta_{2}, \ldots, \delta_{a+b-i-1}, \\
& \left.(1-t) \delta_{a+b-i}+t \ell_{i+1}, \quad \ell_{i}, \ell_{i-1}, \ldots, \ell_{1}\right\},
\end{aligned}
$$

which emphasizes the fact that just one equation in the homotopy varies with $t$. Let $T_{b-1}$ and $T_{i}$ be the set of endpoints of the homotopy paths of $H_{X, b-1}=0$ and $H_{i+1, i}=0$, resp. For $i=\kappa, \ldots, b-1$, sort these into two distinct sets:

- $\widehat{\mathcal{S}}_{i}=T_{i} \cap \Delta$ is a witness point superset for dimension $i$; and

- $N_{i}=T_{i} \backslash \Delta$ is the subset of so-called non-solutions.

Then, $N_{i}$ is the set of starting points for homotopy $H_{i, i-1}$. By Item 3 above, the points $N_{i}$ are nonsingular, which makes the paths easy to track. Note that by Item 2 all the points in $\widehat{\mathcal{S}}_{i}$ are solutions on components of dimension at least $i$ and they remain so as we descend the cascade. These points cannot lead to lower dimensional components, which is why we do not need to use them as start points for the succeeding homotopies.

Several phenomena contribute to the efficiency of a cascade approach, as follows.

- The number of homotopy paths decreases by $\#\left(\widehat{\mathcal{S}}_{i}\right)$ at each stage of the cascade. Also, the endpoints of any paths that diverge to infinity are eliminated as start points for subsequent stages.

- By dropping $\widehat{\mathcal{S}}_{i}$ from the set of start points for $H_{i, i-1}$, the number of junk points in $\widehat{\mathcal{S}}_{i-1}$ is reduced, thus reducing computation in the next phase where we winnow true witness point sets from the witness point supersets.

- The system $\left(D_{i+1}, L_{i+1}\right)$ differs from system $\left(D_{i}, L_{i}\right)$ in only one equation, which tends to result in shorter paths than those for the homotopy $H_{X, i}$, thereby reducing the time spent tracking paths.

\subsection{Intrinsic homotopies}

The homotopies $H_{X, i}$ and $H_{i+1, i}$ are said to be extrinsic, because the linear equations $\left(D_{i}, L_{i}\right)$ appear explicitly. An intrinsic homotopy eliminates these equations by working instead with a set of basis vectors for the associated linear spaces. Since these basis vectors are computed just once when forming the intrinsic homotopy, this reduces the size of the linear system solved in each step of path-tracking to the dimension of the intrinsic linear space, i.e., to the number of basis vectors. However, this reduction comes at a cost: the Jacobian matrix for the new intrinsic variables must be evaluated using the chain rule. We will evaluate this cost trade-off after describing the intrinsic homotopy.

Both $H_{X, i}$ and $H_{i+1, i}$ are of the form

$$
H(x, y, t)=\left\{F_{A}(x), F_{B}(y), t G_{1}(x, y)+(1-t) G_{0}(x, y)\right\},
$$

where $G_{1}, G_{0}: \mathbb{C}^{2 N} \rightarrow \mathbb{C}^{a+b}$ are systems of linear equations. Moreover, these systems have been chosen general enough that they are full-rank systems. Accordingly, the solution sets can be fully characterized by linear functions $\Psi_{1}, \Psi_{0}: \mathbb{C}^{2 N-(a+b)} \rightarrow \mathbb{C}^{2 N}$. That is, there exists $u_{0}, \ldots, u_{2 N-(a+b)}, v_{0}, \ldots, v_{2 N-(a+b)} \in \mathbb{C}^{2 N}$ such that 


$$
\begin{gathered}
\mathcal{V}\left(G_{0}\right)=\left\{u_{0}+\sum_{j=1}^{2 N-(a+b)} u_{j} z_{j} \mid z=\left(z_{1}, \ldots, z_{2 N-(a+b)}\right) \in \mathbb{C}^{2 N-(a+b)}\right\} \text { and } \\
\mathcal{V}\left(G_{1}\right)=\left\{v_{0}+\sum_{j=1}^{2 N-(a+b)} v_{j} z_{j} \mid z=\left(z_{1}, \ldots, z_{2 N-(a+b)}\right) \in \mathbb{C}^{2 N-(a+b)}\right\} .
\end{gathered}
$$

We may rewrite this in matrix form as $\Psi_{1}(z)=v_{0}+V z$, where $V=\left[\begin{array}{lll}v_{1} & \cdots & v_{2 N-(a+b)}\end{array}\right]$. Similarly, we may write $\Psi_{0}(z)=u_{0}+U z$ where $U=\left[\begin{array}{lll}u_{1} & \cdots & u_{2 N-(a+b)}\end{array}\right]$.

Let $\Phi(z, t)=t \Psi_{1}(z)+(1-t) \Psi_{0}(z)$. Then, defining $\pi_{1}:(x, y) \mapsto(x)$ and $\pi_{2}:(x, y) \mapsto(y)$, the intrinsic homotopies we use are:

$$
\mathcal{H}(z, t)=\left\{F_{A}\left(\pi_{1}(\Phi(z, t))\right), F_{B}\left(\pi_{2}(\Phi(z, t))\right)\right\} .
$$

If we had that

$$
H\left(\pi_{1}(\Phi(z, t)), \pi_{2}(\Phi(z, t)), t\right)=\mathcal{H}(z, t),
$$

then the two homotopies would have the same paths and the justification for the transformation from extrinsic to intrinsic form would be immediate. Unfortunately, this is not the case, so we must provide another form of proof. Note that in [24, some rather involved linear algebra was used to formulate intrinsic homotopies that had exactly the same solution paths as their extrinsic counterparts. We take a different approach here.

Since at any fixed value of $t \in \mathbb{C}$ the set $Q_{t}=\left\{\Phi(z, t) \mid z \in \mathbb{C}^{2 N-(a+b)}\right\} \subset \mathbb{C}^{2 N}$ is a linear space, one could construct a continuous path $\Gamma(t) \subset\langle x, y, 1\rangle^{a+b}$ (a path in the space of $(a+b)$ linear equations) such that $Q_{t}=\mathcal{V}(\Gamma(t))$. The validity of an intrinsic homotopy using $\Phi(z, t)$ is proved by showing that $\Gamma(t)$ forms a valid extrinsic homotopy.

Consider first the homotopy $H_{X, b-1}(x, y, t)$ and its corresponding intrinsic homotopy, which we denote $\mathcal{H}_{X, b-1}(z, t)$. For the solution paths of $\mathcal{H}_{X, b-1}(z, t)=0$ to have the desired properties, all we need is that the corresponding path $\Gamma(t)$ is general in $\langle x, y, 1\rangle^{a+b}$ for $t \neq 0$, landing in $\langle x-y\rangle^{a+1} \times\langle x, y, 1\rangle^{b-1}$ at $t=0$. Clearly, $\Gamma(t)$ is in $\langle x, y, 1\rangle^{a+b}$ for all $t$ and due to the way $\Phi(z, t)$ is constructed, we know that $\Gamma(1)$ and $\Gamma(0)$ satisfy the starting and ending conditions. To guarantee that $\Gamma(t)$ stays general as $t$ goes from 1 to 0 , we send it along the real-one-dimensional path

$$
t=p(\tau):=\frac{\gamma \tau}{1+(\gamma-1) \tau}, \quad \tau \in[0,1]
$$

for a generic $\gamma \in \mathbb{C}$. Since the parameter space is Cartesian, Lemma 7.1.3 ("Gamma Trick") of 31 guarantees that the path in parameter space is general for all $\gamma \in \mathbb{C}$ except for a finite number of one-real-dimensional rays from the origin.

For each homotopy $H_{i+1, i}(x, y, t)$, the corresponding intrinsic homotopy will be denoted by $\mathcal{H}_{i+1, i}(z, t)$. For each of these, we need that the corresponding path $\Gamma(t)$ is generic in $\langle x-y\rangle^{a+b-(i+1)} \times\langle x, y, 1\rangle^{i+1}$ for $t \neq 0$ and lands in $\langle x-y\rangle^{a+b-i} \times\langle x, y, 1\rangle^{i}$ at $t=0$. Again, the construction of $\Phi(z, t)$ satisfies the conditions at $t=1$ and $t=0$, but it remains to be shown that the path remains in the appropriate subspace for the whole path. But this is clearly true, because the equations $\left\{\delta_{1}, \delta_{2}, \ldots, \delta_{a+b-(i+1)}\right\}$ are common to both $G_{1}(x, y)$ and $G_{0}(x, y)$. These are homogeneous linear functions, so for example,

$$
\begin{aligned}
& \delta_{1}(\Phi(z, t))=\delta_{1}\left(t \Psi_{1}(z)+(1-t) \Psi_{0}(z)\right) \\
& \quad=t \delta_{1}\left(\Psi_{1}(z)\right)+(1-t) \delta_{1}\left(\Psi_{0}(z)\right)=t \cdot 0+(1-t) \cdot 0=0 .
\end{aligned}
$$

To make sure the one-real-dimensional path from $t=1$ to $t=0$ path is generic, we again use the "gamma trick" of (15). 


\subsection{Cost trade-off}

We compare the costs of using an intrinsic homotopy versus an extrinsic one based on the number of multiplications needed to perform one step of Newton's method, which dominates the cost of path tracking. The intrinsic homotopies are evaluated in a straight-line fashion. In particular, one first computes $(x, y)=\Phi(z, t)$ and then $F_{A}(x)$ and $F_{B}(y)$. Both the extrinsic and intrinsic homotopies evaluate $F_{A}(x)$ and $F_{B}(y)$, so we may neglect that cost. The extrinsic homotopy works with $2 N$ variables, $(x, y) \in \mathbb{C}^{2 N}$, whereas the intrinsic one works with $2 N-(a+b)$ variables, $z \in \mathbb{C}^{2 N-(a+b)}$. To execute a step of Newton's method, we need to evaluate the Jacobian matrices $J_{(x, y)} H(x, y, t)$ and $J_{z} \mathcal{H}(z, t)$ and perform one linear solve. The Jacobian for the extrinsic homotopy evaluates $J_{x} F_{A}(x)$ and $J_{y} F_{B}(y)$, while the intrinsic one uses these post-multiplied by matrices $\partial x / \partial z$ and $\partial y / \partial z$, respectively. So the intrinsic homotopy incurs an extra $(2 N-(a+b))^{2} N$ multiplications. Solving using standard Gaussian elimination with partial pivoting costs $(2 N-(a+b))^{3} / 3+O\left((2 N-(a+b))^{2}\right)$ for the intrinsic homotopy and $(2 N)^{3} / 3+O\left(N^{2}\right)$ for the extrinsic one. If we define $\alpha$ such that $a+b=\alpha N$, then comparing only the cubic terms, the break even point occurs when $(2-\alpha)^{2}(5-\alpha)=8$. Accordingly, ignoring the lower-order terms, the intrinsic homotopy is less expensive when $\alpha \geq 0.645$, i.e., when $a+b \geq 0.645 N$.

\section{From witness point sets to witness sets}

After computing the witness point supersets, $\widehat{\mathcal{S}}_{i}$ using the algorithm described in Section 3 , we want to eliminate junk points to obtain the pure $i$-dimensional witness point set, $\mathcal{S}_{i} \subset \mathcal{S}_{i}$ and then decompose this into witness point sets, $\mathcal{S}_{i j}$, for the irreducible components, $\mathcal{Z}_{i j}$ of $X \cap \Delta$. Then, we have witness point sets $S_{i j}$ for the irreducible components $Z_{i j}$ of $A \cap B$ using the projection $S_{i j}=\pi_{\Delta}\left(\mathcal{S}_{i j}\right)$. We also need to find a witness system, $F_{i j}$, for each $Z_{i j}$, such that $Z_{i j}$ is an irreducible component of $\mathcal{V}\left(F_{i j}\right)$. The following subsection shows how isosingular sets are relevant to these goals.

\subsection{Isosingularity of witness sets}

Theorem 4.1.1 (Main Result) Let $F_{A}: \mathbb{C}^{N} \rightarrow \mathbb{C}^{n_{a}}$ and $F_{B}: \mathbb{C}^{N} \rightarrow \mathbb{C}^{n_{b}}$ be polynomial systems with irreducible components $A \subset \mathcal{V}\left(F_{A}\right)$ and $B \subset \mathcal{V}\left(F_{B}\right)$. Define $F(x, y)=\left\{F_{A}(x), F_{B}(y)\right\}$ and $\Delta=\left\{(z, z) \mid z \in \mathbb{C}^{N}\right\}$. Suppose that $Z$ is an irreducible component of $A \cap B$ and $\mathcal{Z}=\pi_{\Delta}^{-1}(Z)$. Then, there is a nonempty Zariski open set $U \subset Z$ such that for all $u \in U$, $\mathcal{Z}$ is an irreducible component of $\operatorname{Iso}_{F}((u, u)) \cap \Delta$.

Proof. Since $\mathcal{Z}$ is an irreducible algebraic set contained in $\mathcal{V}(F)$, there is a nonempty Zariski open set $\mathcal{U} \subset \mathcal{Z}$ such that for every $(u, u) \in \mathcal{U}, \mathcal{Z}$ and $(u, u)$ have the same deflation sequence with respect to $F$. Define $U=\pi_{\Delta}(\mathcal{U})$, which is a nonempty Zariski open subset of $Z$, and fix $u \in U$. Since $(u, u) \in \mathcal{U}$, we know that $\mathcal{Z} \subset \operatorname{Iso}_{F}((u, u))$ which yields

$$
\mathcal{Z} \subset \operatorname{Iso}_{F}((u, u)) \cap \Delta .
$$

Thus, there exists an irreducible component $\mathcal{W} \subset \operatorname{Iso}_{F}((z, z)) \cap \Delta$ such that $\mathcal{Z} \subset \mathcal{W}$. Consider $W=\pi_{\Delta}(\mathcal{W})$. We clearly have $Z \subset W$ and, since $W$ is isomorphic to $\mathcal{W}, W$ is an irreducible algebraic set. 
Since $A \times B \subset \mathcal{V}(F)$ is an irreducible component with $(u, u) \in A \times B$, we know that $\operatorname{Iso}_{F}((u, u)) \subset A \times B$. This yields

$$
\mathcal{Z} \subset \mathcal{W} \subset \operatorname{Iso}_{F}((u, u)) \cap \Delta \subset(A \times B) \cap \Delta
$$

and hence $Z \subset W \subset A \cap B$. Since $Z$ is an irreducible component of $A \cap B$ and $W$ is an irreducible algebraic set, we must have $Z=W$. Therefore, $\mathcal{Z}=\mathcal{W}$, and hence $\mathcal{Z}$ is an irreducible component of $\operatorname{Iso}_{F}((u, u)) \cap \Delta$.

The immediate upshot of Theorem 4.1.1 is that it gives a way of finding the polynomial systems that complete the witness sets for irreducible components. We codify the procedure in the following corollary.

Corollary 4.1.2 Suppose $F$ and $Z$ are as in Theorem 4.1.1. For any $z \in Z$, let $\mathcal{G}_{z}(x, y)=$ $D^{k^{*}}(F(x, y),(z, z))$, where $k^{*}$ is the isosingular stabilization index of $(z, z)$, and let $G_{z}(x)=$ $\mathcal{G}_{z}(x, x)$. Then, there is a nonempty Zariski open set $U \subset Z$ such that for all $u \in U, Z$ is an irreducible component of $\mathcal{V}\left(G_{u}\right)$.

Proof. By Theorem 2.0.3. Item 2. $\operatorname{Iso}_{F}((z, z))$ is an irreducible component of $\mathcal{V}\left(\mathcal{G}_{z}\right)$. By Theorem 4.1.1 $\mathcal{Z}=\pi_{\Delta}^{-1}(Z)$ is an irreducible component of $\operatorname{Iso}_{F}((u, u)) \cap \Delta$. The corollary follows by restricting $\mathcal{G}_{z}(x, y)$ to the diagonal, $\Delta$, and then using the isomorphism $Z=\pi_{\Delta}(\mathcal{Z})$.

We note that the assumptions made in Section 3 , namely that $A$ and $B$ were generically reduced and the dimensions of $A$ and $B$ were compatible with the number of polynomials in $F_{A}$ and $F_{B}$, respectively, were only made for computational convenience. In particular, these assumptions yielded homotopies that were square and solutions paths that were nonsingular for $t=p(\tau), 0<\tau \leq 1$. These assumptions are not needed to apply Theorem 4.1.1 meaning that we could use one set of polynomial systems to perform the computations in Section 3 and use the original set of polynomial systems when applying Theorem 4.1.1.

Example 4.1.3 Reconsider Example 1.0.2 where $F_{A}(x, y, z)=x$ and $F_{B}(x, y, z)=x y$ with irreducible components $A=\{(0, y, z) \mid y, z \in \mathbb{C}\}$ and $B=\{(x, 0, z) \mid x, z \in \mathbb{C}\}$, respectively. In light of Corollary 4.1 .2 , consider

$$
F\left(x_{1}, x_{2}, x_{3}, y_{1}, y_{2}, y_{3}\right)=\left[\begin{array}{c}
F_{A}\left(x_{1}, x_{2}, x_{3}\right) \\
F_{B}\left(y_{1}, y_{2}, y_{3}\right)
\end{array}\right]=\left[\begin{array}{c}
x_{1} \\
y_{1} y_{2}
\end{array}\right] \text { and } Z=A \cap B=\{(0,0, \alpha) \mid \alpha \in \mathbb{C}\} .
$$

Since, for every $a \in \mathbb{C}, \operatorname{Iso}_{F}((0,0, a, 0,0, a))=\{(0, \alpha, \beta, 0,0, \gamma) \mid \alpha, \beta, \gamma \in \mathbb{C}\}$, we have $U=Z$ and $\operatorname{Iso}_{F}((0,0, a, 0,0, a)) \cap \Delta=\{(0,0, \alpha, 0,0, \alpha) \mid \alpha \in \mathbb{C}\}$. In particular, for any $a \in \mathbb{C}$,

$$
\mathcal{G}_{(0,0, a)}(x, y)=\left[\begin{array}{c}
x_{1} \\
y_{1} y_{2} \\
y_{1} \\
y_{2}
\end{array}\right] \quad \text { and } \quad G_{(0,0, a)}(x)=\left[\begin{array}{c}
x_{1} \\
x_{1} x_{2} \\
x_{1} \\
x_{2}
\end{array}\right] .
$$

Clearly, $Z=A \cap B$ is a generically reduced, irreducible component of $\mathcal{V}\left(G_{(0,0, a)}\right)$. We may replace $G_{(0,0, a)}$ with $G^{\prime}(x)=\left[\begin{array}{ll}x_{1} & x_{2}\end{array}\right]$. 


\subsection{Completing the decomposition}

At the end of the diagonal cascade, we have witness point supersets $\widehat{\mathcal{S}}_{i}, i=\kappa, \ldots, b-1$. Consider a point $(z, z) \in \widehat{\mathcal{S}}_{i}$. It is either:

- a generic point of some $i$-dimensional irreducible component, say $\mathcal{Z}_{i j}$, of $X \cap \Delta$; or

- a junk point, i.e., a point on some component(s) of dimension $k>i$.

In the first case, the genericity of $(z, z)$ is assured, with probability one, by the random choice of the linear space $\mathcal{L}_{i}$. Points of this type belong in the witness point set for $\mathcal{Z}_{i j}$. In the second case, there is no guarantee that $x$ is a generic member of any higher-dimensional irreducible component: in fact, it often will be in the singular set of one or more such components.

Consider the following preliminary decomposition procedure for dimension $i$. It consists of the following steps.

\section{Preliminary Decomposition:}

1. For each point $(z, z) \in \widehat{\mathcal{S}}_{i}$ use the determinantal deflation procedure to find the system $\mathcal{G}(x, y)=D^{k^{*}}(F(x, y),(z, z))$ where $k^{*}$ is the isosingular stabilization index. In so doing, we also find the point's first deflation sequence $d_{k}(F,(z, z)), k=0,1, \ldots, k^{*}$.

2. Let $G(x):=\mathcal{G}(x, x)$. For each point also find $G^{*}(x)=D^{\ell^{*}}(G(x), z)$, where $\ell^{*}$ is the isosingular stabilization index, thereby determining the point's second deflation sequence $d_{k}(G, z), k=0,1, \ldots, \ell^{*}$.

3. Sort all the points of $\widehat{\mathcal{S}}_{i}$ into subsets of points that have the same first and second deflation sequences. Each subset has an associated $G^{*}(x)$.

Since true witness points are generic on their associated irreducible component, all the witness points on the same irreducible component must fall into the same subset after this preliminary decomposition. Moreover, Corollary 4.1.2 tells us that $G(x)$ is a witness system for that component, while by Theorem 2.0.3. Items 3 and $4 . G^{*}(x)$ meets the stronger condition that the irreducible component is generically reduced with respect to it. Furthermore, by Theorem 2.0.3. Item 2, we have that the stabilization value, $d_{\ell^{*}}$, of the second deflation sequence is the dimension of the irreducible component.

Remark 4.2.1 Due to the last observation above, any point in $\widehat{\mathcal{S}}_{i}$ whose deflation sequence (first or second) dips below the current dimension, i.e., $d_{k}<i$, must be junk and can be immediately eliminated. This may happen because although a junk point lies on some higher dimensional component(s), it may be a singular point such that its isosingular set is of lower dimension.

Remark 4.2.2 Our experience is that the second deflation sequence ends with $\ell^{*}=0$, but we have no proof that this will always be the case.

Remark 4.2.3 Each point $(z, z) \in \widehat{\mathcal{S}}_{i}$ lies in $\mathcal{V}\left(L_{i}(x, y)\right) \cap \Delta$, hence its projection $z=\pi_{\Delta}(z, z)$ lies in the linear space $\mathcal{V}\left(L_{i}(x, x)\right) \subset \mathbb{C}^{N}$. The genericity of $L_{i}(x, y)$ implies that $L_{i, \Delta}(x):=$ $L_{i}(x, x)$ is also generic, so $L_{i, \Delta}(x)$ serves as the linear space for witness sets in the original coordinate space, $\mathbb{C}^{N}$. For notational simplicity, we use $L_{i}(x, x)$ in writing witness sets below.

Remark 4.2.4 In the special case where a point $(z, z) \in \widehat{\mathcal{S}}_{i}$ has $k^{*}=\ell^{*}=0$, the point $z$ is a nonsingular solution of $\left\{F(x, x), L_{i}(x, x)\right\}=0$. Thus, $z$ does not lie in a higher-dimensional 
component and must be a true witness point. Moreover, $F(x, x)=\left\{F_{A}(x), F_{B}(x)\right\}$ serves as a witness system for the component witnessed by $z$. Note that the subset of all such points in $\widehat{\mathcal{S}}_{i}$ forms a witness point set for a pure $i$-dimensional algebraic set, but it may comprise a union of irreducible components that still needs to be factored to obtain the irreducible decomposition.

These facts justify the following procedure for eliminating all junk points and performing a partial numerical irreducible decomposition of $A \cap B$.

\section{Partial Decomposition:}

1. Find the top dimension: If all $\widehat{\mathcal{S}}_{i}=\emptyset, i=\kappa, \ldots, b-1$, then $A \cap B=\emptyset$, and we are done. Otherwise, there must be a largest $i$, call it $i^{*}$, among these such that $\widehat{\mathcal{S}}_{i}$ is not empty. Then, $i^{*}=\operatorname{dim}(A \cap B)$. Since there are no components of higher dimension, all the points in $\widehat{\mathcal{S}}_{i^{*}}$ are true witness points; that is, $\mathcal{S}_{i^{*}}=\widehat{\mathcal{S}}_{i^{*}}$ and $S_{i^{*}}=\pi_{\Delta}\left(\mathcal{S}_{i^{*}}\right)$.

2. Reduce the top dimension: Apply the preliminary decomposition to $\mathcal{S}_{i^{*}}$. Note that since there are no higher dimensional components, each reduction must end with $d_{\ell^{*}}=i^{*}$. Using the witness systems provided by the preliminary decomposition, form the witness sets for the top dimension: $\widetilde{W}_{i^{*} j}=\left\{G_{i^{*} j}^{*}(x), L_{i^{*}}(x, x), P_{i^{*} j}\right\}, j=1, \ldots, m_{i^{*}}$.

3. Descend the dimensions: For $i=\left(i^{*}-1\right), \ldots, \kappa$ do the following.

(a) Split off nonsingular points: Remove the points conforming to the conditions of Remark 4.2 .4 from $\widehat{\mathcal{S}}_{i}$ and place their projections under $\pi_{\Delta}$ in a witness set $\widetilde{W}_{i 0}=$ $\left\{F(x, x), L_{i}(x, x), P_{i 0}\right\}$.

(b) Eliminate junk: For each remaining point $(z, z) \in \widehat{\mathcal{S}}_{i}$ perform homotopy membership tests from each witness set $\widetilde{W}_{\tilde{\imath}, j}$, all $\tilde{\imath}>i, j=0, \ldots, m_{\tilde{\imath}}$, stopping whenever a membership is detected. If $z$ is a member of any of these higher-dimensional components, it is a junk point, and so is $(z, z)$, meaning we discard it. Otherwise, we retain $(z, z)$ in $\mathcal{S}_{i}$.

(c) Reduce singular points: Apply the preliminary decomposition procedure above to $\mathcal{S}_{i}$, obtaining a coarse subdivision of $S_{i}$ into $m_{i}$ distinct sets, say $S_{i}=\cup_{j=1}^{m_{i}} P_{i j}$, each with a corresponding witness system $G_{i j}^{*}(x)$. Form the witness sets $\widetilde{W}_{i j}=$ $\left\{G_{i j}^{*}(x), L_{i}(x, x), P_{i j}\right\}, j=1, \ldots, m_{i}$.

Final Decomposition: After the foregoing procedure, any of the witness sets $\widetilde{W}_{i j}, i=\kappa, \ldots, i^{*}$, $j=0,1, \ldots, m_{i}$, might represent more than one irreducible component. To complete the numerical irreducible decomposition, one may apply monodromy and trace tests as described in 28, 29. After this is done, one has a complete numerical irreducible decomposition into witness sets $W_{i j}$ corresponding to the decomposition of $A \cap B$ into irreducible components $Z_{i j}$ :

$$
A \cap B=\bigcup_{i=\kappa}^{i^{*}} Z_{i}, \quad Z_{i}=\bigcup_{j=0}^{n_{i}} Z_{i j}, \quad n_{i} \geq m_{i} .
$$

Remark 4.2.5 In the Partial Decomposition procedure, the order of Steps 3b and 3c could be reversed. In that case, Remark 4.2.1 may be useful in eliminating some junk points. The choice of which order is most efficient for eliminating junk points depends on the number of higher dimensional witness points (known) and the stabilization indices, $k^{*}$ and $\ell^{*}$, for the test point (unknown at the outset). Both procedures run to completion for true witness points, so the order does not matter for those. 


\section{$5 \quad$ Examples}

We first present a basic example in $\mathbb{C}^{4}$ and then report on an example arising in kinematics. Both of these examples used Bertini v1.3.1 [3] for the homotopy computations.

\subsection{A basic example}

Consider the polynomial systems $F_{A}, F_{B}: \mathbb{C}^{4} \rightarrow \mathbb{C}^{2}$ defined by

$$
F_{A}(x, y, z, w)=\left[\begin{array}{c}
x z^{2}+5 x^{2}+x w-7 y^{2}-x \\
y z w-3 x^{2}+2 y^{2}+y z-2 y
\end{array}\right] \text { and } F_{B}(x, y, z, w)=\left[\begin{array}{c}
x z^{2}-3 x^{2}+x w+11 y^{2}-x \\
y z w+9 x^{2}-5 y^{2}+y z-2 y
\end{array}\right] .
$$

It is easy to verify that $\mathcal{V}\left(F_{A}\right)$ and $\mathcal{V}\left(F_{B}\right)$ each decompose into the union of the plane $\mathcal{V}(x, y)$ and a degree 7 surface, which we denote $A$ and $B$, respectively, with $A \not \subset B$ and $B \not \subset A$.

Starting with witness sets for $A$ and $B$, the first step is to track 49 paths using the homotopy $H_{X, 1}$ defined by 10$)$. This yields the sets $\widehat{\mathcal{S}}_{1}$ consisting of 4 points and $N_{1}$ consisting of 40 points. The remaining five paths diverged to infinity. Since $\widehat{\mathcal{S}}_{1} \neq \emptyset$, we know $\operatorname{dim}(A \cap B)=1$ and that each point in $\widehat{\mathcal{S}}_{1}$ is a witness point, i.e., $\mathcal{S}_{1}=\widehat{\mathcal{S}}_{1}$.

Starting with the points in $N_{1}$, the next step is to track 40 paths using the homotopy $H_{1,0}$ defined by 12 . This yields the set $\widehat{\mathcal{S}}_{0}$ consisting of seven points, three of which are the endpoint of a unique path and the other four are the endpoint of seven paths. The remaining 19 paths diverged to infinity.

We now proceed to the preliminary decomposition of $\mathcal{S}_{1}$. Each of the four points in $\mathcal{S}_{1}$ has a first deflation sequence of $6,2,2, \ldots$ with $k^{*}=1$ and a second deflation sequence of $1,1, \ldots$ with $\ell^{*}=0$. Hence, each point has the same associated system $G^{*}$, which is constructed as follows. Let $F: \mathbb{C}^{8} \rightarrow \mathbb{C}^{4}$ be constructed from $F_{A}$ and $F_{B}$ as in Theorem 4.1.1 and fix $\alpha \in \mathcal{S}_{1}$. Since dnull $(F,(\alpha, \alpha))=6$, let $\mathcal{G}$ be the system obtained by appending the $3 \times 3$ determinants of the Jacobian of $F$ to $F$. There are $\left(\begin{array}{l}4 \\ 3\end{array}\right) \cdot\left(\begin{array}{l}8 \\ 3\end{array}\right)=224$ such determinants, 128 of which are zero and only 48 of the remaining 96 are independent. Since $\ell^{*}=0$, we have $G^{*}(x)=G(x)=\mathcal{G}(x, x)$.

Using $G^{*}$, we can now perform a homotopy membership test to eliminate junk from the witness point superset $\widehat{\mathcal{S}}_{0}$. This test found that all seven points in $\widehat{\mathcal{S}}_{0}$ lie on a one-dimensional component in $A \cap B$ yielding that there are no zero-dimensional components.

In the final decomposition phase, we used the trace test on the four points in $\mathcal{S}_{1}$ yielding two irreducible curves of degree 2 , say $C_{1}$ and $C_{2}$. With $G^{*}$ as the witness system, we may now form a witness set for each of $C_{1}$ and $C_{2}$, using the linear system $L(x):=L_{1}(x, x)$ from 10 . along with the corresponding witness points.

Since the final decomposition phase produces witness sets for $C_{1}$ and $C_{2}$, these can be used for further computations on those sets. In particular, we can proceed to compute $C_{1} \cap C_{2}$, which turns out to consist of three isolated points.

\subsection{Griffis-Duffy platform of type II}

Griffis-Duffy platforms are special cases of Stewart-Gough platforms, a kind of parallel-link mechanism in which six extensible legs with spherical joints at each end support a moving platform above a stationary base. In a general configuration, the extensions of the six legs can be actuated to move the platform to obtain any translation and orientation within a sixdimensional subset of $S E(3)$, and when the leg lengths are locked, the mechanism becomes rigid. This is useful, for example, as the motion base in a flight simulator, and the mechanism finds other applications in industrial robotics. 
The Griffis-Duffy special case of the Stewart-Gough platform occurs when the spherical joints on the base form a triangle, with the center of three of the joints at the vertices and one each of the other three along the edges, and the same is true for the moving platform. Moreover, a leg attached to a vertex in the base is attached to an edge in the moving platform and vice versa, with the connections proceeding in sequence around the triangles. The forward kinematics of these mechanisms, that is, the determination of the location of the moving plate given the leg lengths, is simpler than for the general Stewart-Gough platform, which motivated a patent of the device by its eponymous inventors [6. Subsequently, Husty and Karger [11 noticed that if the base and upper plate are both equilateral and the joints on the edges are placed at the midpoints, then the mechanism becomes architecturally singular. This means that no matter what location the upper plate is placed in when the leg lengths are locked, the mechanism still moves along a curve. This is the type I variant of special Griffis-Duffy platforms. A subset of these, the type II variant, occurs when the upper and lower triangles are congruent and the leg lengths are all equal, in which case the motion curve factors into several irreducible components. In [11, symbolic methods were used to determine the irreducible decomposition, which was confirmed with minor corrections by the numerical irreducible decomposition computed in [22]. It is interesting to note that when the leg lengths of the type II mechanism are equal to the altitude of the triangles, the mechanism can fold into a configuration where the triangles coincide. The real motion curves of this foldable Griffis-Duffy platform were computed as an example in [17.

We shall consider the type II Griffis-Duffy platform as the squared leg length, $\ell$, varies. The polynomial system describing the kinematics of the mechanism depends upon $\ell \in \mathbb{C}$ and $\left[e_{1}, \ldots, e_{4}, g_{1}, \ldots, g_{4}\right] \in \mathbb{P}^{7}$. We dehomogenize by working on a random coordinate patch defined by $P\left(e_{1}, \ldots, e_{4}, g_{1}, \ldots, g_{4}\right)=0$ where $P: \mathbb{C}^{8} \rightarrow \mathbb{C}$ is an affine random linear polynomial. We first consider the polynomial system $\mathcal{F}=\left\{f_{1}, \ldots, f_{7}, P\right\}$, where each $f_{i}$ is defined in the Appendix A as a system on $\mathbb{C}^{9}$. The irreducible decomposition of $\mathcal{V}(\mathcal{F})$ consists of a not physically meaningful 3-fold and 17 two-dimensional components: twelve planes, one sextic, three surfaces of degree 11, and one of degree 13. Among these, there are two surfaces of degree 11 which do not lie in a coordinate hyperplane, which we denote as $A$ and $B$.

Since $f_{6} \in\left\langle f_{1}, \ldots, f_{5}\right\rangle$, we used the polynomial system $F_{A}=F_{B}=\left\{f_{1}, \ldots, f_{5}, f_{7}, P\right\}$, which consists of 7 polynomials in 9 variables, to perform the homotopy computations. Starting with witness point sets for $A$ and $B$ and tracking the 121 paths of the homotopy $H_{X, 1}$ defined by 10), one obtains $\widehat{\mathcal{S}}_{1}$ containing five points and $N_{1}$ containing 114 points. The remaining 2 paths diverged to infinity. Since $\widehat{\mathcal{S}}_{1} \neq \emptyset$, we know $\operatorname{dim}(A \cap B)=1$ and that each point in $\widehat{\mathcal{S}}_{1}$ is a witness point, i.e., $\mathcal{S}_{1}=\widehat{\mathcal{S}}_{1}$.

Using the homotopy $H_{1,0}$ defined by $(12)$ and tracking the 114 paths emanating from the points in $N_{1}$, one obtains $\widehat{\mathcal{S}}_{0}$ containing six points and $N_{0}$ containing 66 points. The remaining 42 paths diverged to infinity.

We now turn to the preliminary decomposition for $\mathcal{S}_{1}=\widehat{\mathcal{S}}_{1}$. The first two terms in the first deflation sequence is 8,8 for two points in $\mathcal{S}_{1}$ and 8,2 for the other three points. This implies that $A \cap B$ must contain at least two irreducible curves. Since a membership test using $\mathcal{F}$ shows that the two points with first two terms 8,8 are contained in the not physically meaningful 3 -fold, we will only consider the other three points.

The first deflation sequence of these three points is $8,2,2, \ldots$ with $k^{*}=1$ and a second deflation sequence of $1,1, \ldots$ with $\ell^{*}=0$. The trace test yields that these three points form a witness point set for an irreducible curve $C$ of degree 3 . Membership tests show that each point in $\widehat{\mathcal{S}}_{0}$ lies in $C$, so there are no isolated solutions.

Let $\pi: \mathbb{C}^{9} \rightarrow \mathbb{C}$ be the map defined by $\pi\left(\ell, e_{1}, \ldots, e_{4}, g_{1}, \ldots, g_{4}\right)=\ell$. The approach of [7] 
implies that $\pi$ is generically a two-to-one map from $C$ to $\mathbb{C}$ with critical points at $\ell=1 / 3$ and $\ell=3$. The real Stewart-Gough platforms in $C$ correspond to the set $\pi^{-1}([1 / 3,3]) \cap C$. In Figure 1. we display the configuration of the mechanism for one of the two points in $\pi^{-1}(\ell) \cap C$ for selected values of $\ell$ between $1 / 3$ and 3 . The lighter shaded triangle is the stationary base and the darker one is the moving platform. As $\ell$ increases, the two triangles remain coplanar with their centers coincident, and the moving platform rotates $180^{\circ}$ in the clockwise direction. The other point of $\pi^{-1}(\ell) \cap C$, not illustrated, has the same starting and ending configuration, but rotates in the counter-clockwise direction.

Each surface, $A$ and $B$, is the union taken as $\ell$ varies of the self-motion curves obtained with the legs locked at length $\sqrt{\ell}$. As such, the configurations shown in Figure 1 represent the points where a self-motion curve from $A$ meets a self-motion curve from $B$.

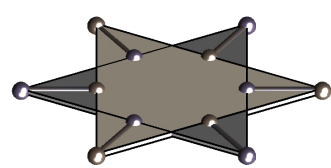

$\ell=1 / 3$

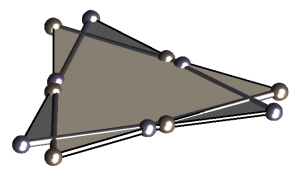

$\ell=4 / 3$

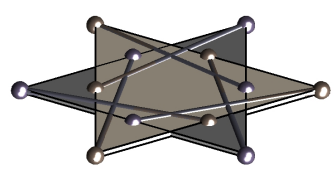

$\ell=7 / 3$

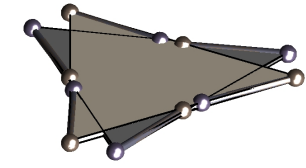

$\ell=2 / 3$

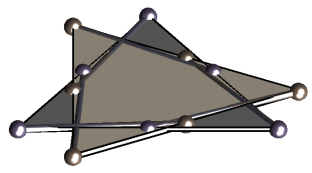

$\ell=5 / 3$

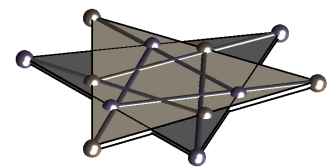

$\ell=8 / 3$

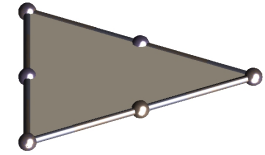

$\ell=1$

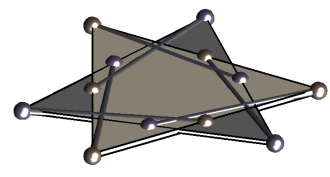

$\ell=2$

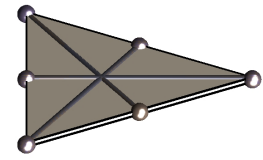

$\ell=3$

Figure 1: Griffis-Duffy type II platforms on the curve $C$ for various $\ell$ 


\section{Discussion}

In the presentation of this paper, we have exclusively relied on the determinantal form of strong deflation. As compared to other forms presented in [10, this avoids introducing auxiliary variables. This simplifies intersections after deflation - otherwise one must intersect projections that drop away the auxiliary variables - and all other things being equal, having fewer variables is generally beneficial for reducing computation. However, determinantal strong deflation has the downside that it may introduce a large number of equations, as the number of minors can be large. In practice, it appears that only a few of the minors are independent, so it may be hoped that symbolic methods can be developed for reducing the number of minors that are introduced at any stage. If this hope goes unrealized, in some situations it may be better to use the other forms of deflation. This is an open issue that deserves further study.

\section{Conclusion}

The completion of the diagonal homotopy brings to numerical algebraic geometry the full capability of performing all Boolean operations on algebraic sets, thereby extending the reach of the methods to a full treatment of constructible algebraic sets. The key step in this completion is contained in Theorem 4.1.1, which applies isosingular theory [10] to the diagonal homotopy framework first established in [23].

\section{References}

[1] V.I. Arnol'd. Singularities of smooth mappings. Russian Math. Surveys 23, 1-43, 1968.

[2] D.J. Bates, J.D. Hauenstein, C. Peterson, and A.J. Sommese. A numerical local dimension test for points on the solution set of a system of polynomial equations. SIAM J. Numer. Anal. 47(5), 3608-3623, 2009.

[3] D.J. Bates, J.D. Hauenstein, A.J. Sommese, and C.W. Wampler. Bertini: Software for Numerical Algebraic Geometry. Available at www.nd.edu/ sommese/bertini

[4] J. Boardman. Singularities of differentiable maps. Inst. Hautes Études Sci. Publ. Math. $33,21-57,1967$.

[5] B.H. Dayton and Z. Zeng. Computing the multiplicity structure in solving polynomial systems. In ISSAC'05, pp. 116-123. ACM, New York, 2005.

[6] M. Griffis and J. Duffy. Method and apparatus for controlling geometrically simple parallel mechanisms with distictive connections. US Patent 5,179,525, 1993.

[7] J.D. Hauenstein and A.J. Sommese. Witness sets of projections. Appl. Math. Comput. 217(7), 3349-3354, 2010.

[8] J.D. Hauenstein, A.J. Sommese, and C.W. Wampler. Regeneration homotopies for solving systems of polynomials. Math. Comp. 80, 345-377, 2011.

[9] J.D. Hauenstein, A.J. Sommese, and C.W. Wampler. Regenerative cascade homotopies for solving polynomial systems. Appl. Math. Comp., 218(4), 1240-1246, 2011. 
[10] J.D. Hauenstein and C.W. Wampler. Isosingular sets and deflation. Preprint, 2011. Available at www.math.tamu.edu/ jhauenst/preprints.

[11] M.L. Husty and A. Karger. Self-motions of Griffis-Duffy type parallel manipulators. In Proc. IEEE Int. Conf. Robotics and Automation, San Francisco, CA, April 2000.

[12] G. LeCerf. Quadratic Newton iteration for systems with multiplicity. Found. Comp. Math. 2(3), 247-293, 2002.

[13] A. Leykin, J. Verschelde, and A. Zhao. Higher-order deflation for polynomial systems with isolated singular solutions. In Algorithms in Algebraic Geometry, volume 146 of IMA Vol. Math. Appl., pp. 79-97. Springer, New York, 2008.

[14] A. Leykin, J. Verschelde, and A. Zhao. Newton's method with deflation for isolated singularities of polynomial systems. Theor. Comp. Sci. 359, 111-122, 2006.

[15] T.-Y. Li. Numerical solution of multivariate polynomial systems by homotopy continuation methods. Acta Numerica, 6, 399-436, 1997.

[16] T.-Y. Li. Numerical solution of polynomial systems by homotopy continuation methods. In Handbook of Numerical Analysis, Vol. XI, pp. 209-304, North-Holland, Amsterdam, 2003.

[17] Y. Lu, D.J. Bates, A.J. Sommese, and C.W. Wampler. Finding all Real Points of a Complex Curve. In Proc. Midwest Algebra, Geometry and Its Interactions Conf., Contemporary Mathematics, AMS, 448, 183-205, 2007.

[18] A.P. Morgan. Solving Polynomial Systems Using Continuation for Engineering and Scientific Problems. Prentice Hall Inc., Englewood Cliffs, NJ, 1987.

[19] T. Ojika. Modified deflation algorithm for the solution of singular problems. I. A system of nonlinear algebraic equations. J. Math. Anal. Appl. 123, 199-221, 1987.

[20] T. Ojika, S. Watanabe, and T. Mitsui. Deflation algorithm for the multiple roots of a system of nonlinear equations. J. Math. Anal. Appl. 96, 463-479, 1983.

[21] A.J. Sommese and J. Verschelde. Numerical homotopies to compute generic points on positive dimensional algebraic sets. J. Complexity, 16(3), 572-602, 2000.

[22] A.J. Sommese, J. Verschelde, and C.W. Wampler. Advances in polynomial continuation for solving problems in kinematics. ASME J. Mech. Design 126(2), 262-268, 2004.

[23] A.J. Sommese, J. Verschelde, and C.W. Wampler. Homotopies for intersecting solution components of polynomial systems. SIAM J. Numer. Anal. 42(4), 1552-1571, 2004.

[24] A.J. Sommese, J. Verschelde, and C.W. Wampler. An intrinsic homotopy for intersecting algebraic varieties. J. Complexity, 21(4), 593-608, 2005.

[25] A.J. Sommese, J. Verschelde, and C.W. Wampler. A method for tracking singular paths with application to the numerical irreducible decomposition. In Algebraic Geometry, a Volume in Memory of Paolo Francia, ed. by M.C. Beltrametti, et al., pp. 329-345, W. de Gruyter, 2002. 
[26] A.J. Sommese, J. Verschelde and C.W. Wampler. Numerical decomposition of the solution sets of polynomial systems into irreducible components. SIAM J. Numer. Anal. 38(6), 2022-2046, 2001.

[27] A.J. Sommese, J. Verschelde, and C.W. Wampler. Numerical irreducible decomposition using projections from points on the components. In Symbolic computation: solving equations in algebra, geometry, and engineering (South Hadley, MA, 2000), volume 286 of Contemp. Math., 37-51. Amer. Math. Soc., Providence, RI, 2001.

[28] A.J. Sommese, J. Verschelde, and C.W. Wampler. Symmetric functions applied to decomposing solution sets of polynomial systems. SIAM J. Numer. Anal., 40(6), 2026-2046, 2002.

[29] A.J. Sommese, J. Verschelde, and C.W. Wampler. Using monodromy to decompose solution sets of polynomial systems into irreducible components. In Applications of algebraic geometry to coding theory, physics and computation (Eilat, 2001), volume 36 of NATO Sci. Ser. II Math. Phys. Chem., 297-315. Kluwer Acad. Publ., Dordrecht, 2001.

[30] A.J. Sommese and C.W. Wampler. Numerical algebraic geometry, In The Mathematics of Numerical Analysis (Park City, UT, 1995), Lectures in Appl. Math., 32, 749-763, AMS, Providence, RI, 1996.

[31] A.J. Sommese and C.W. Wampler. The Numerical Solution of Systems of Polynomials Arising in Engineering and Science. World Scientific Press, Singapore, 2005.

[32] C.W. Wampler and A.J. Sommese. Numerical algebraic geometry and algebraic kinematics. Acta Numerica, 20, 469-567, 2011.

[33] Z. Zeng. The closedness subspace method for computing the multiplicity structures of a polynomial system. Contemp. Math., 496, 347-362, AMS, Providence, RI, 2009.

\section{A Stewart-Gough platform system}

The polynomials for the Stewart-Gough platform, which are presented using the variables $\ell, e_{1}$, $e_{2}, e_{3}, e_{4}, g_{1}, g_{2}, g_{3}, g_{4}$, are as follows. If $r=\sqrt{3}, E=e_{1}^{2}+e_{2}^{2}+e_{3}^{2}+e_{4}^{2}$, and $G=g_{1}^{2}+g_{2}^{2}+g_{3}^{2}+g_{4}^{2}$, the polynomial system is $\left\{f_{1}, \ldots, f_{7}\right\}$ defined as

$$
\begin{aligned}
& f_{1}= 7 e_{1}^{2} / 3-4 r e_{1} e_{2} / 3+e_{2}^{2}+e_{3}^{2} / 3+3 e_{4}^{2}-r e_{1} g_{3} / 3+3 e_{1} g_{4}-e_{2} g_{3}-r e_{2} g_{4}+r e_{3} g_{1} / 3+e_{3} g_{2} \\
& \quad-3 e_{4} g_{1}+r e_{4} g_{2}+G-\ell E \\
& f_{2}= 7 e_{1}^{2} / 3+4 r e_{1} e_{2} / 3+e_{2}^{2}+e_{3}^{2} / 3+3 e_{4}^{2}-r e_{1} g_{3} / 3+3 e_{1} g_{4}+e_{2} g_{3}+r e_{2} g_{4}+r e_{3} g_{1} / 3-e_{3} g_{2} \\
& \quad-3 e_{4} g_{1}-r e_{4} g_{2}+G-\ell E \\
& f_{3}=e_{1}^{2} / 3+3 e_{2}^{2}+e_{3}^{2} / 3+3 e_{4}^{2}+2 r e_{1} g_{3} / 3+2 r e_{2} g_{4}-2 r e_{3} g_{1} / 3-2 r e_{4} g_{2}+G-\ell E \\
& f_{4}=7 e_{1}^{2} / 3-4 r e_{1} e_{2} / 3+e_{2}^{2}+e_{3}^{2} / 3+3 e_{4}^{2}-r e_{1} g_{3} / 3-3 e_{1} g_{4}-e_{2} g_{3}+r e_{2} g_{4}+r e_{3} g_{1} / 3+e_{3} g_{2} \\
& \quad+3 e_{4} g_{1}-r e_{4} g_{2}+G-\ell E \\
& f_{5}=7 e_{1}^{2} / 3+4 r e_{1} e_{2} / 3+e_{2}^{2}+e_{3}^{2} / 3+3 e_{4}^{2}-r e_{1} g_{3} / 3-3 e_{1} g_{4}+e_{2} g_{3}-r e_{2} g_{4}+r e_{3} g_{1} / 3-e_{3} g_{2} \\
& \quad+3 e_{4} g_{1}+r e_{4} g_{2}+G-\ell E \\
& f_{6}=e_{1}^{2} / 3+3 e_{2}^{2}+e_{3}^{2} / 3+3 e_{4}^{2}+2 r e_{1} g_{3} / 3-2 r e_{2} g_{4}-2 r e_{3} g_{1} / 3+2 r e_{4} g_{2}+G-\ell E \\
& f_{7}=e_{1} g_{1}+e_{2} g_{2}+e_{3} g_{3}+e_{4} g_{4}
\end{aligned}
$$

